\title{
STABILITY OF NONLINEAR SYSTEMS UNDER CONSTANTLY ACTING PERTURBATIONS
}

\author{
XINZHI LIU \\ Department of Applied Mathematics \\ Iniversity of Waterloo \\ Waterloo, Ontario N2L 3G1, Cianada

\section{S. SIVASUNDARAM} \\ Department of Mathematics and Physical Sciences \\ Embry-Riddle Aeronautical University \\ Daytona Beach. FL 32114, U.S.A. \\ (Received September 9, 1991)
}

\begin{abstract}
In this paper, we investigate total stability, attractivity and uniform stability in terms of two measures of nonlinear differential systems under constant perturbations. Some sufficient conditions are obtained using Lyapunov's direct method. An example is also worked out.
\end{abstract}

KEY WORDS AND PHRASES. Stability, perturbation, Lyapunov function, two measures. 1980 AMS (MOS) SUBJECT CLASSIFICATION CODE. 34D20, 34D30, 34D10.

\section{INTRODUCTION}

When we model a physical system by means of a differential equation, it is not generally possible to take into account all the causes which determine the evolution. In other words, we have to admit that there are small perturbations permanently acting which cannot be accurately estimated consequently the validity of the description of the evolution, as given by a corresponding solution of the differential equation, requires that this solution be "stable" not only with respect to the small perturbations of the initial conditions, but also with respect to the perturbations, small in a suitable sense, of the right hand side of the equation. This kind of stability is called total stability, which we shall define in the next section.

There are several different concepts of stability studied in the literature, such as eventual stability, partial stability, conditional stability, etc. To unify these varieties of stability notions and to offer a general basis for investigation, it is convenient to introduce stability in terms of two different measures. Following Movchan [4], Salvadori [5] has successfully developed the theory of stability in terms of two measures. In the recent years much work has been done using two measures. See $[2,3]$ and references therein.

In this paper we investigate the total stability, attractivity and uniform stability of perturbed systems in terms of two measures. In view of the generality of the present approach, our results improve and include some of the earlier findings and may be suitable for many applications.

\section{PRELIMINARIES}

Consider the differential system

$$
x^{\prime}=f(t, x), \quad x\left(t_{0}\right)=x_{0}
$$

and the perturbed differential system

$$
x^{\prime}=f(t, x)+R(t, x), \quad x\left(t_{0}\right)=x_{0},
$$

where $f, R \in C\left[R_{+} \times R^{n}, R^{n}\right], \quad R(t, x)$ is a perturbation term relative to unperturbed system $(2.1)$

Let us begin by defining the following class of functions for future use. 


$$
\begin{gathered}
K^{\prime}=\left\{a \in C^{\prime}\left[R_{+}, R_{+}\right]: a(u) \quad \text { is strictly increasing and } a(0)=0\right\} ; \\
\Gamma=\left\{h \in C^{C}\left[R_{+} \times R^{N}, R_{+}\right], \quad \text { inf } h(t, x)=0\right\} .
\end{gathered}
$$

Definition 2.1 Let $h_{0}, h \in \mathrm{I}$, then we say that $h_{0}$ is uniformly finer than $h$ if there exists a $\delta>0$ and a function $\Phi \in K$ such that

$$
h_{0}(t, x)<\delta \quad \text { implies } h(t, x) \leq \Phi\left(h_{0}(t, x)\right) .
$$

Definition 2.2 Let $V \epsilon C\left[R_{+} \times R^{N}, R_{+}\right]$and $h_{0}, h \in \Gamma$ then $V(t, x)$ is said to be

(i) $h$-positive definite if there exist a $\rho>0$ and a function $b \in K$ such that $h(t, x)<\rho$ implies $b(t(t, x)) \leq V(t, x)$.

(ii) $h_{0}$ - decrescent if there exist a $\delta>0$ and a function $a \in K$ such that

$$
h_{0}(t, x)<\delta \text { implies } V(t, x) \leq a\left(h_{0}(t, x)\right) .
$$

Let $h_{0}, h \in \Gamma$. We shall now define the stability concepts for the system (2.1) in terms of two measures $\left(h_{0}, h\right)$. Let $S(h, \rho)=\left[(t, x) \in R_{+} \times R^{n}, h(t, x)<\rho\right]$.

Definition 2.3 The system (2.1) is said to be $\left(h_{0}, h, T_{1}\right)$ - totally stable, if given $\epsilon>0$ and $t_{0} \epsilon R_{+}$, there exist two numbers $\delta_{1}, \delta_{2}>0$ such that $h_{0}\left(t_{0}, x_{0}\right)<\delta_{1}$ and

$$
\|R(t, x)\|<\delta_{2} \quad \text { for } \quad(t, x) \in S(h, \epsilon)
$$

imply $h\left(t, y\left(t, t_{0}, x_{0}\right)\right)<\epsilon, \quad t \geq t_{0}, \quad$ where $y\left(t, t_{0}, x_{0}\right)$ is any solution of the perturbed system (2.2).

Definition 2.4 The system (2.1) is said to be $\left(h_{0}, h, T_{2}\right)$ - totally stable, if for every $\epsilon>0$, $t_{0} \in R^{+}$and $T>0$ there exist two positive numbers $\delta_{1}=\delta_{1}(\epsilon)$ and $\delta_{2}=\delta_{2}(\epsilon)$ such that for every solution $y(t)=y\left(t, t_{0}, x_{0}\right)$ of $(2.2)$ the inequality $h(t, y(t))<\epsilon, \quad t \geq t_{0}$ satisfies, provided that $h_{0}\left(t_{0}, x_{0}\right)<\delta_{1}, \quad\|R(t, x)\| \leq \mu(t)$ for $h(t, x) \leq \epsilon$ and $\int_{t}^{t+T} \mu(s) d s<\delta_{2}$.

Definition 2.5 The system (2.1) is said to be $\left(h_{0}, h\right)$ - attractive if given $t_{0} \in R_{+}$, there exist a positive constant $\delta_{0}=\delta\left(t_{0}\right)$ such that $h_{0}\left(t_{0}, x_{0}\right)<\delta_{0}$ implies $\left.\lim _{t \rightarrow \infty} h(t, x)\right)=0$.

We need the following known results [1] for our discussion.

Lemma 2.1 Let $g \in C\left[R_{+} \times R, R\right]$ and $r(t)=r\left(t, t_{0}, x_{0}\right)$ be maximal solution of

$$
u^{\prime}=g(t, u), u\left(t_{0}\right)=u_{0} \geq 0
$$

existing on $J$. Suppose that $m \in\left[R_{+}, R_{+}\right]$and $D m(t) \leq g(t, m(t)), \quad t \in J$ where $D$ is any fixed Dini derivative. Then $m\left(t_{0}\right) \leq u_{0}$ implies $m(t) \leq r(t), t \in J$.

\section{MAIN RESULTS}

In this section we shall investigate the stability and attractivity properties of the differential system.

THEOREM 3.1 Assume that

(i) $h_{0}, h \in \Gamma$ and $h_{0}$ is uniformly finer than $h$.

(ii) $V \in C^{1}\left[R_{+} \times R^{n}, R_{+}\right], V(t, x)$ is $h$-positive definite, $h_{0}$-decrescent and

$$
V_{2.2}^{\prime}(t, x) \leq-C\left(h_{0}(t, x)\right), \quad(t, x) \in S(h, \rho), \quad C \epsilon K .
$$

(iii) $\|V(t, x)-V(t, y)\| \leq M\|x-y\|, \quad(t, x),(t, y) \in S(h, \rho)$ and $M>0$.

Then the system $(2.1)$ is $\left(h_{0}, h, T_{1}\right)$ - totally stable. 
PROOF: Let us write $V_{2}^{\prime}(t, y)$, the time derivative of $V$ along the solutions of the perturbed system (2.2). Then it follows from (ii) and (iii) that

$$
V_{22}^{\prime}(t, y) \leq-C\left(h_{0}(t, y)\right)+M\|R(t, y)\|,(t, y) \in S(h, \rho)
$$

Since $V(t, x)$ is $h$-positive definite and $h_{0}$-decrescent, there exist constants $\rho_{0} \in(0, \rho), \delta_{0}>0$ and functions $a, b \in K$ such that

$$
V(t, x) \leq a\left(h_{0}(t, x)\right) \quad \text { if } \quad h_{0}(t, x)<\delta_{0}
$$

and

$$
b(h(t, x)) \leq V(t, x) \quad \text { whenever } \quad h(t, x)<\rho_{0} .
$$

Let $\epsilon \in\left(0, \rho_{0}\right)$ be given. Choose $\delta_{1} \in\left(0, \delta_{0}\right)$ such that

$$
a\left(\delta_{1}\right)<b(\epsilon) \quad \text { and } \quad h(t, x)<\epsilon \quad \text { if } \quad h_{0}(t, x)<\delta
$$

because of the assumptions on $a, b$ and condition (i).

For $K \in(0,1)$, choose $\delta_{2}=K \frac{C\left(\delta_{1}\right)}{M}$. Let $t_{0} \in R_{+}$and $y(t)=y\left(t, t_{0}, x_{0}\right)$ be a solution of (2.2) we claim that $h\left(t_{0}, x_{0}\right)<\delta$, and $\|R(t, y)\|<\delta_{2}$ for $(t, y) \in S(h, \epsilon)$ implies

$$
h(t, y(t))<\epsilon, \quad t \geq t_{0} .
$$

If this is not true, there would exist a solution $y(t)=y\left(t, t_{0}, x_{0}\right)$ of $(2.2)$ with $h_{0}\left(t_{0}, x_{0}\right)<\delta_{1}$ and $t_{2}>t_{1}>t_{0}$ such that

$$
h_{0}\left(t_{1}, y\left(t_{1}\right)\right)=\delta_{1}, \quad h\left(t_{2}, y\left(t_{2}\right)\right)=\epsilon,(t, y(t)) \in S(h, \epsilon) \cap S^{c}\left(h_{0}, \delta_{1}\right)
$$

and

$$
\|R(t, y(t))\|<\delta_{2}, \quad t \in\left[t_{1}, t_{2}\right) .
$$

Then it follows from (3.1) and (3.6) that

$$
V^{\prime}(t, y(t)) \leq-C\left(\delta_{1}\right)+M K \frac{C\left(\delta_{1}\right)}{M}<0, \quad t_{1} \leq t \leq t_{2} .
$$

which implies by (3.2)-(3.4) that

$$
b(\epsilon) \leq V\left(t_{2}, y\left(t_{2}\right)\right) \leq V\left(t_{1}, y\left(t_{1}\right)\right) \leq a\left(\delta_{1}\right)<b(\epsilon) .
$$

This contradiction shows that (3.5) is true and thus the system $(2.1)$ is $\left(h_{0}, h, T_{1}\right)$ - totally stable which completes the proof of the theorem.

THEOREM 3.2 In addition to the assumption of theorem 3.1, suppose further that there exist a constant $\sigma>0$ such that $h(t, x)<\sigma$ implies

$$
\lim _{t \rightarrow \infty} R(t, y)=0
$$

uniformly in $y$.

Then the system $(2.2)$ is $\left(h_{0}, h\right)$-attractive.

PROOF: Because of $\left(h_{0}, h\right)$ - total stability of system $(2.1)$, setting $\epsilon=\sigma_{0}=\min \left\{\rho_{0}, \sigma\right\}$, there exists constants $\delta_{10}>0$ and $\delta_{20}>0$ such that $h_{0}\left(t_{0}, x_{0}\right)<\delta_{10}$ and $\|R(t, x)\|<\delta_{20}$ for $(t, x) \in S\left(h, \sigma_{0}\right)$ implies

$$
h(t, y(t))<\sigma_{0}, \quad t \geq t_{0},
$$

where $y(t)=y\left(t, t_{0}, x_{0}\right)$ is any solution of $(2.2)$.

Let $\eta \in\left(0, \sigma_{0}\right)$ and $\delta_{1}=\delta_{1}(\eta), \quad \delta_{2}=\delta_{2}(\eta)$ be chosen as in the definition 2.3. Let $\sigma_{2}^{*}=\min \left\{\delta_{2}, \frac{C\left(\delta_{1}\right)}{M}\right\}$, it follows from (3.7) that there exist $T_{1}=T\left(t_{0}, x_{0}\right)>0$ such that 


$$
\|R(t, y(t))\|<\delta_{2}^{*}, \quad t \geq T+t_{0}
$$

To show $\left(h_{0}, h\right)$-attractivity of $(2.2)$. it is cnough to prove that there exists a $T=T\left(t_{0}, x_{0}\right)>0$ such that for some $t^{*} \in\left[t_{0}, T+t_{0}\right]$

$$
h_{0}\left(t^{*}, x\left(t^{*}\right)\right)<\delta_{1} \quad \text { and } \quad\|R(t, y(t))\|<\delta_{2}^{*}, t \geq t^{*} .
$$

Choose

$$
T=\frac{4 a\left(h_{0}\left(t_{0}+T_{1}, y\left(t_{0}+T_{1}\right)\right)\right)}{C\left(\delta_{1}\right)}+T_{1} .
$$

Then if for $t_{0}+T_{1} \leq t \leq t_{0}+T, \quad(t, y(t)) \in S\left(h, \sigma_{0}\right) \cap S^{c}\left(h_{0}, \delta_{1}\right), \quad$ we get by (3.1) and (3.2)

$$
V^{\prime}(t, y(t)) \leq-\frac{C\left(\delta_{1}\right)}{2}, \quad t_{0}+T_{1} \leq t \leq t_{0}+T .
$$

which implies

$$
V\left(t_{0}+T, y\left(t_{0}+T\right)\right) \leq a\left(h_{0}\left(t_{0}+T_{1}, y\left(t_{0}+T_{1}\right)\right)\right)-\frac{C\left(\delta_{1}\right)}{2}\left(T-T_{1}\right)<0 .
$$

This contradiction shows the existence of $t^{*}$ and it follows from $\left(h_{0}, h, T_{1}\right)$ - total stability of $(2.1)$ that the system $(2.2)$ is $\left(h_{0}, h\right)$ - attractive, which completes the proof of the theorem.

The next result is on $\left(h_{0}, h, T_{2}\right)$ - stability.

THEOREM 3.3 Assume that

(i) $h_{0}, h \in \Gamma$ and $h_{0}$ is uniformly finer than $h$.

(ii) $V \in C^{1}\left[R_{+} \times R^{n}, R_{+}\right], V(t, x)$ is $h$-positive definite $V(t, x)$ is $h_{0}$-decrescent and

$$
V_{2.1}^{\prime}(t, x) \leq-C(V(t, x)), \quad(t, x) \epsilon S(h, \rho), \quad C \in K .
$$

(iii) $\|V(t, x)-V(x, y)\| \leq M \quad\|x-y\|, \quad(t, x),(t, y) \in S(h, \rho)$ and $M>0$.

Then the system $(2.1)$ is $\left(h_{0}, h, T_{2}\right)$ - totally stable.

PROOF: Using the relations (3.1) and (3.2) we choose $\delta_{1}=\delta_{1}(\epsilon)$ such that

$$
a\left(\delta_{1}\right)<b(\epsilon) .
$$

Let $h_{0}\left(t_{0}, x_{0}\right)<\delta_{1}, \quad m(t)=V(t, y(t))$, where $y(t)=y\left(t, t_{0}, x_{0}\right)$ is a solution of (2.2). Hence $m\left(t_{0}\right)<a\left(\delta_{1}\right)<b(\epsilon)$. We claim $m(t)<b(\epsilon), t \geq t_{0}$. If this is not true, then there exist a $t_{1}>t_{0}$ such that $m\left(t_{1}\right)=b(\epsilon)$ and $m(t) \leq b(\epsilon)$ for $t_{0} \leq t_{1}$ which implies

$$
h(t, y(t)) \leq \epsilon<\rho, \quad t_{0} \leq y \leq t_{1}
$$

Let $t_{1}-t_{0}=T$ and choose

$$
\delta_{2}=\delta_{2}(\epsilon)<b(\epsilon)-J^{-1}\left\{J\left(a\left(\delta_{1}\right)\right)\right\} / M
$$

where

$$
J(u)-J\left(u_{0}\right)=\int_{u_{0}}^{u} \frac{d s}{C(s)}, \quad J(u)=\int_{0}^{u} \frac{d S}{C(s)} \quad \text { if } \quad \int_{0}^{u} \frac{d s}{C(s)}<\infty
$$

Otherwise $J(u)=\int_{\delta}^{u} \frac{d s}{C(s)}$ for some small constant $\delta>0$, and $J^{-1}$ is the inverse function of $J$.

From (ii) and (iii) we have

$$
D^{+} V(t, y(t)) \leq-C(V(t, y(t)))+M\|R(t, y(t))\|
$$

for $t \in\left[t_{0}, t_{1}\right]$. 
Now define $\lambda(t)=I^{\prime}(t, y(t))-\gamma(t)$. where

$$
\vartheta(t)=.1 / \int_{t,}^{t}\|R(s, y(\cdot))\| d s .
$$

We obtain

$$
1)^{+} \lambda(t) \leq-\left(^{\prime}(\lambda(1))\right.
$$

using the monotonic character of $C^{\prime}(u)$ and the fact $\lambda(l) \geq V(t, y(t))$, which implies, by Lemma 2.1 , that

$$
\lambda(t) \leq J^{-1}\left[J\left(V\left(t_{0}, x_{0}\right)\right)-\left(t-t_{0}\right)\right], \quad t \in\left[t_{0}, t_{1}\right\}
$$

Noting that the maximal solution of $u^{\prime}=-C(u), u\left(t_{0}\right)=V\left(t_{0}, x_{0}\right)$, is just the right hand side of (3.13), thus it follows

$$
V(t, y(t)) \leq J^{-1}\left[J\left(1 \cdot\left(t_{0}, x_{0}\right)\right)-\left(t-t_{0}\right)\right]+\gamma(t) . \quad t \in\left[t_{0}, t_{1}\right] .
$$

Now using the facts that $h(t, y(t)) \leq c$ for $t_{0} \leq t \leq t_{0}+T, V\left(t_{0}, x_{0}\right)<a\left(\delta_{1}\right),\|R(t, y(t))\| \leq \mu(t)$, $\int_{t}^{t+T} \mu(s) d s<\delta_{2}$ and relations (3.10), (3.12), we derive the inequality

$$
b(\epsilon) \leq V\left(t_{0}+T, y\left(t_{0}+T\right)\right) \leq J^{-1}\left[J\left(a\left(\delta_{1}\right)\right)-T\right]+M \delta_{2}<b(\epsilon),
$$

which is a contradiction. Thus $m(t)<b(\epsilon), t \geq t_{0}$, which implies $\left(h_{0}, h, T_{2}\right)$-total stability of (2.1). This completes the proof of the theorem.

In the previous theorems, in order to prove total stability properties of $(2.2)$ we assumed the uniform asymptotic stability properties of (2.1) (the unperturbed system). In the following theorem we prove $\left(h_{0}, h\right)$ - stability of (2.2) under weaker assumptions on (2.1) by avoiding using norm on the perturbed term.

\section{THEOREM 3.4 Assume that}

(i) $h_{0}, h \in \Gamma$ and $h_{0}$ is uniformly finer than $h$.

(ii) $V \in C^{1}\left[R_{+} \times R^{n}, R_{+}\right], V(t, x)$ is $h$-positive definite, $h_{0}$-decrescent and

$$
V_{2.1}^{\prime}(t, x) \leq 0, \quad(t, x) \in S(h, \rho) .
$$

(iii) $\frac{\partial V(t, x)}{\partial x} \cdot R(t, x) \leq \ell(t) V(t, x), \quad(t, x) \in S(h, \rho)$, where $\ell(t) \in \mathcal{L}^{1}$ and $\exp \left[\int_{t_{1}}^{t_{2}} \ell(s) d s\right] \leq M, M>0$.

Then the system $(2.2)$ is $\left(h_{0}, h\right)$-uniformly stable.

PROOF: Let us write $V_{2.2}^{\prime}(t, y)$, the time derivative of $V$ along the solutions of the perturbed system (2.2). Then it follows from (ii) and (iv) that

$$
V_{2.2}^{\prime}(t, y) \leq V_{2.1}^{\prime}(t, y)+\frac{\partial V(t, x)}{\partial x} \cdot R(t, x) \leq \ell(t) V(t, y),(t, y) \in S(h, \rho)
$$

Since $V(t, x)$ is $h$-positive definite and $h_{0}$-decrescent, there exist constants $\rho_{0} \in(0, \rho), \delta_{0}>0$ and functions $a, b \in K$ such that

$$
V(t, x) \leq a\left(h_{0}(t, x)\right) \quad \text { if } \quad h_{0}(t, x)<\delta_{0}
$$

and

$$
b(h(t, x)) \leq V(t, x) \quad \text { whenever } \quad h(t, x)<\rho_{0}
$$

Let $\epsilon \in\left(0, \rho_{0}\right)$ be given. Because of the assumptions of $a, b$ and condition (i) we can choose $\delta_{1} \in\left(0, \delta_{0}\right)$ such that

$$
M a\left(\delta_{1}\right)<b(\epsilon) \text { and } h(t, x)<\epsilon \text { if } h_{0}(t, x)<\delta_{1} .
$$


Let $t_{0} \in R_{+}$and $y(t)=y\left(t, t_{1}, x_{0}\right)$ be a solution of $(2.2)$. We claim that $h\left(t_{0}, x_{0}\right)<\delta_{1}$ implies

$$
h(t, y(t))<c . \quad t \geq t_{0} \quad \text { for } \quad(t, y) \in S(h, c)
$$

If this is not true, there would exist a solution $y(t)=y\left(t, t_{0}, x_{0}\right)$ of $(2.2)$ with $h_{0}\left(t_{0}, x_{0}\right)<\delta_{1}$ and $t_{2}>t_{1}>t_{0}$ such that $h_{0}\left({ }_{1}, y\left(t_{1}\right)\right)=\delta_{1}, \quad h\left(t_{2}, y\left(t_{2}\right)\right)=\mathrm{c}$ and

$$
(t, y(t)) c S(h, \in) \cap . \zeta^{\prime}\left(h_{0}, \delta_{1}\right), \quad t \in\left[t_{1}, t_{2}\right),
$$

then it follows from (3.14)

$$
V^{\prime \prime}(t, y(t)) \leq \ell(t) V^{\prime}(t . y(t))
$$

which implies by $(3.15)-(3.18)$

$$
b(\epsilon) \leq V\left(t_{2}, y\left(t_{2}\right) \leq V\left(t_{1}, y\left(t_{1}\right)\right) \exp \left[\int_{t_{1}}^{t_{2}} \ell(s) d s\right] \leq M a\left(\delta_{1}\right)<b(\epsilon) .\right.
$$

This contradiction shows that (3.18) is true, which completes the proof of the theorem.

To conclude our paper, we consider the following example.

EXAMPLE: Consider the differential system

and the perturbed system

$$
\begin{aligned}
& x_{1}^{\prime}=x_{2}+\left(1-x_{1}^{2}-x_{2}^{2}\right) x_{1} c^{-t} \\
& x_{2}^{\prime}=x_{1}+\left(1-x_{1}^{2}-x_{2}^{2}\right) x_{2} \sin x_{1}^{2}
\end{aligned}
$$

$$
\begin{aligned}
& x_{1}^{\prime}=-x_{2}+\left(1-x_{1}^{2}-x_{2}^{2}\right) x_{1} e^{-t}+R_{1}\left(t, x_{1}, x_{2}\right) \\
& x_{2}=x_{1}+1\left(1-x_{1}^{2}-x_{2}^{2}\right) x_{2} \sin x_{1}^{2}+R_{2}\left(t, x_{1}, x_{2}\right)
\end{aligned}
$$

where

$$
R_{1}\left(t, x_{1}, x_{2}\right)=\left(x_{1}^{2}+x_{2}^{2}-1\right) t^{2} \frac{e^{\ell}}{x_{1}}
$$

and

$$
R_{2}\left(t, x_{1}, x_{2}\right)=\left(x_{1}^{2}+x_{2}^{2}-1\right) \frac{e^{-t / 2} \sin t}{x_{2}} .
$$

Let $V(t, x)=\left(x_{1}^{2}+x_{2}^{2}-1\right)^{2}, \quad h_{0}=h=\left|x_{1}^{2}+x_{2}^{2}-1\right|$. Then we see that

$$
h^{2}(t, x) \leq V(t, x) \leq h_{0}^{2}(t, x)
$$

and

$$
\begin{gathered}
V_{4.1}^{\prime}(t, x)=-\left(x_{1}^{2}+x_{2}^{2}-1\right)^{2}\left(x_{1}^{2} e^{-t}+x_{2}^{2} \sin x_{1}^{2}\right) \leq 0, \quad(t, x) \in R_{+} \times R^{2}, \\
\frac{\partial V}{\partial x} \cdot R(t, x)=\frac{\partial V}{\partial x_{1}} R_{1}\left(t, x_{1}, x_{2}\right)+\frac{\partial V}{\partial x_{2}} R_{2}\left(t, x_{1}, x_{2}\right) \leq \ell(t) V(t, x),
\end{gathered}
$$

where $\ell(t)=4\left[t^{2} e^{-t}+\sin t e^{-t / 2}\right]$. Hence by Theorem 3.4, the perturbed system $(4.2)$ is $\left(h_{0}, h\right)$ uniformly stable.

\section{References}

1. LAKSHMIKANTHAM , V. and LEELA, S. Differential and Integral Inequalities, Vol. I, Academic Press, New York (1969).

2. LAKSHMIKANTHAM, V. LEELA, S. and MARTYNYUK, A.A. Stability Analysis of Nonlinear Systems, Marcel Dekker, New York (1989i

3. LAKSHMIKANTHAM, V. and LIU, X.Z. Stability of Nonlinear Systems in Terms of Two Measures, to appear.

4. MOVCHAN, H.A. Stability of Process with Respect to Two Matrices, Prik. Mat. Mech. $24(1960), 988-1001$.

5. SALVADORI, L. Sul problema della stabilita asintotica, Rendı Contı, Acad. No. 2. Lincei 53 (1972), 35-38. 


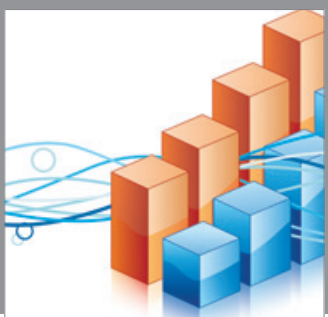

Advances in

Operations Research

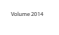

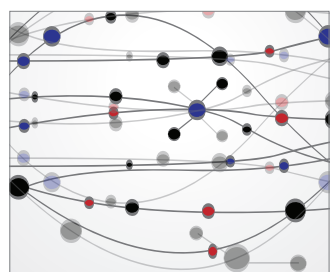

\section{The Scientific} World Journal
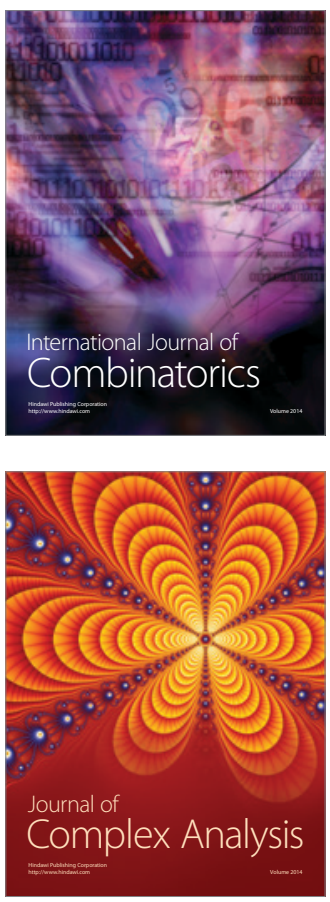

International Journal of

Mathematics and

Mathematical

Sciences
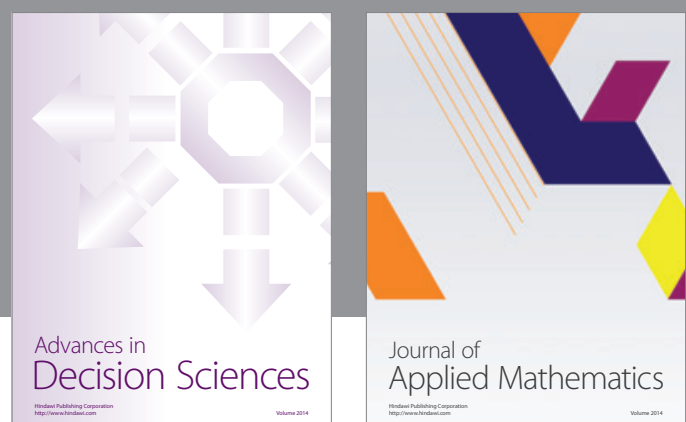

Journal of

Applied Mathematics
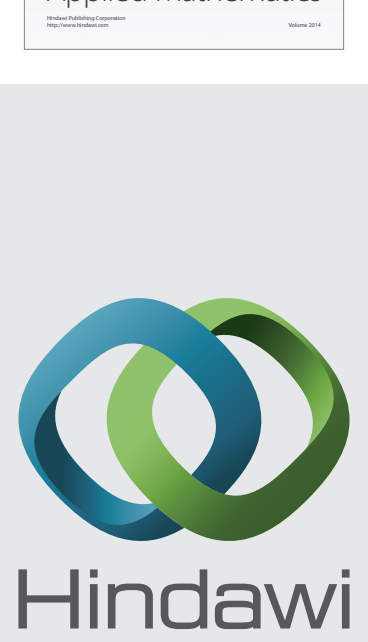

Submit your manuscripts at http://www.hindawi.com
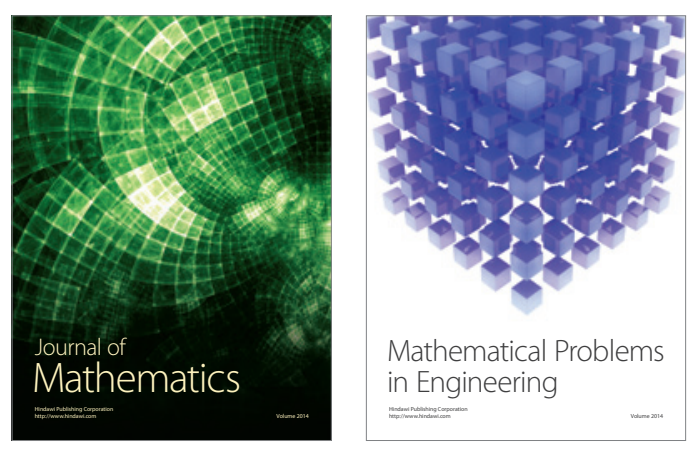

Mathematical Problems in Engineering
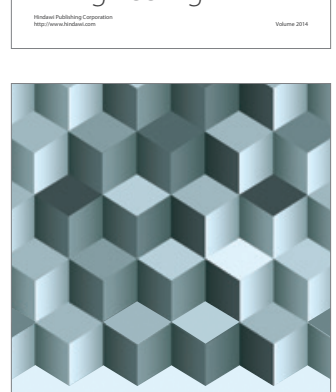

Journal of

Function Spaces
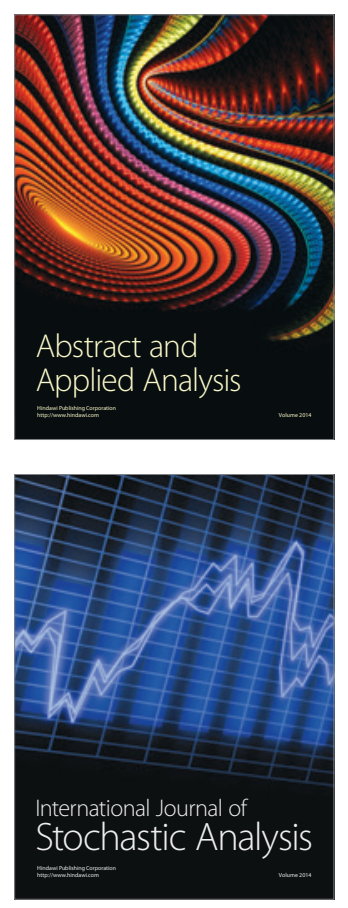

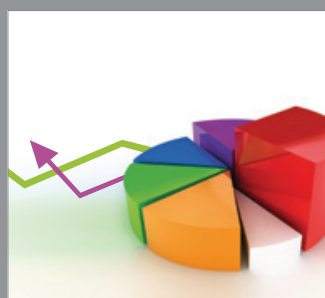

ournal of

Probability and Statistics

Promensencen
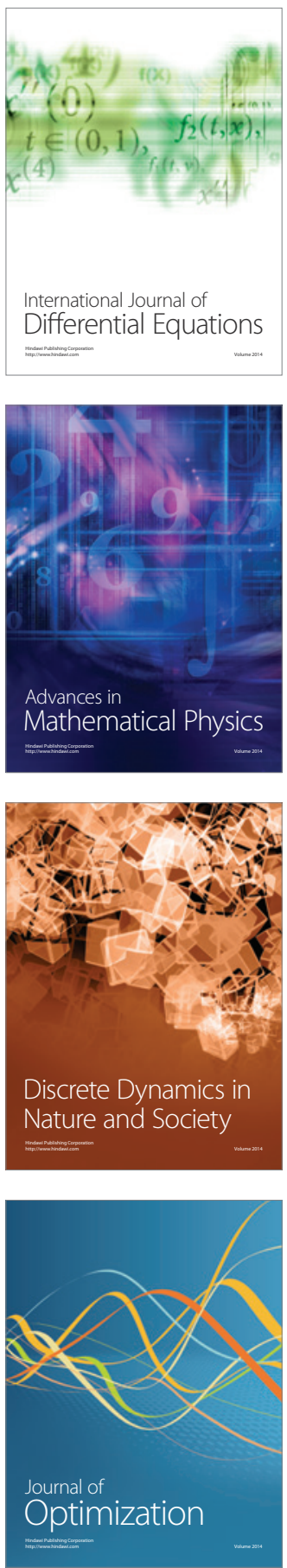